\title{
Feasibility Study of Physician Orders for Life-Sustaining Treatment for Patients with Terminal Cancer
}

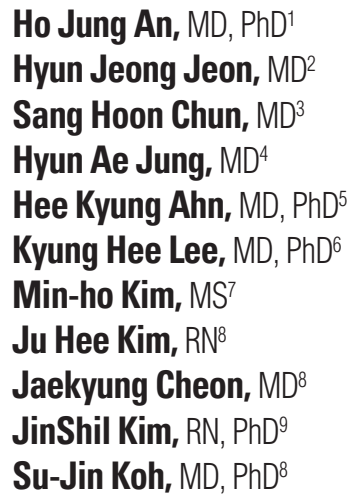

${ }^{1}$ Department of Medical Oncology, St. Vincent's Hospital, College of Medicine, The Catholic University of Korea, Suwon, ${ }^{2}$ Department of Internal Medicine, Seoul Medical Center, Seoul, ${ }^{3}$ Division of Medical Oncology, Department of Internal Medicine, Bucheon St. Mary's Hospital, College of Medicine, The Catholic University of Korea, Bucheon, ${ }^{4}$ Department of Internal Medicine, Hallym University Dongtan Sacred Heart Hospital, Hallym University College of Medicine, Hwaseong, ${ }^{5}$ Division of Medical Oncology, Department of Internal Medicine, Gachon University Gil Medical Center, Incheon, ${ }^{6}$ Department of Internal Medicine, Yeungnam University College of Medicine, Daegu, ${ }^{7}$ Ewha Institute of Convergence Medicine, Ewha Womans University Mokdong Hospital, Seoul, ${ }^{8}$ Department of Hematology and Oncology, Ulsan University Hospital, Ulsan University College of Medicine, Ulsan, ${ }^{9}$ College of Nursing, Gachon University, Incheon, Korea

\author{
Correspondence: Su-Jin Koh, MD, PhD \\ Department of Hematology and Oncology, \\ Ulsan University Hospital, Ulsan University \\ College of Medicine, 877 Bangeojinsunhwando-ro, \\ Dong-gu, Ulsan 4403, Korea \\ Tel: $82-52-250-8978$ \\ Fax: 82-52-250-8978 \\ E-mail: sujinkoh@hanmail.net \\ Received January 3, 2019 \\ Accepted April 16, 2019 \\ Published Online April 18, 2019
}

\begin{abstract}
Purpose
Physician Orders for Life-Sustaining Treatment (POLST) form is a legal document for terminally ill patients to make medical decisions with physicians near the end-of-life. A multicenter prospective study was conducted to evaluate the feasibility of POLST administration in actual oncological practice.
\end{abstract}

\section{Materials and Methods}

Patients with terminal cancer, age $\geq 20$ years, and capable of communicating were eligible. The primary endpoint was the completion rate of POLST. Data about physicians' or patients' barriers were also collected.

\section{Results}

From June to December 2017, 336 patients from seven hospitals were eligible. Median patient age was 66 years (range, 20 to 94 years); $52.7 \%$ were male; and $60.4 \%$ had poor performance status. Primary cancer sites were hepato-pancreato-biliary (26.2\%), lung (23.2\%), and gastrointestinal (19.9\%). Expected survival duration was $10.6 \pm 7.3$ weeks, with $41.2 \%$ receiving hospice care, $37.9 \%$ showing progression after cancer treatment, and the remaining patients were under active treatment $(15.8 \%)$ or initially diagnosed with terminal cancer (5.1\%). POLST forms were introduced to $60.1 \%$ of patients, and $31.3 \%$ signed the form. Physicians' barriers were reluctance of family (49.7\%), lack of rapport (44.8\%), patients' denial of prognosis (34.3\%), lack of time (22.7\%), guilty feelings (21.5\%), and uncertainty about either prognosis (21.0\%) or the right time to discuss POLST (16.6\%). The patients' barriers were the lack of knowledge/understanding of POLST (65.1\%), emotional discomfort (63.5\%), difficulty in decision-making (66.7\%), or denial of prognosis (14.3\%).

\section{Conclusion}

One-third of patients completed POLST forms, and various barriers were identified. To overcome such barriers, social engagement, education, and systematic support might be necessary.
Key words Physician Orders for Life-Sustaining Treatment, Terminal cancer, Feasibility 


\section{Introduction}

In 2009, the Supreme Court of South Korea imposed the withdrawal of a mechanical ventilator used for a female patient, who had been in vegetative status, based on her presumed preferences for end-of-life (EOL) care [1]. This historic judgment aroused public interest regarding discontinuation of futile life-sustaining treatment (LST). An intensive discussion ensued to reach a social consensus for patient autonomy regarding the right to make decisions about EOL treatments. The resulting Life-Sustaining Treatment Decision-Making Act came into full effect in February 2018 [2]. To protect the autonomy and dignity of terminally ill patients, patients can discuss the Physician Orders for Life-Sustaining Treatment (POLST) form with their physicians to decide whether they want to apply or suspend life-prolonging treatment near death. The POLST form is a medical document that mainly pertains to a patient's future care, including EOL care preferences in case they lose the capacity to make decisions. Decision-making primarily addresses preferences for LST. An advance directive (AD) is a legal document written by anyone regardless of his/her illness, and it includes a future medical care plan, living will, or designation of power of attorney [3]. Both POLST and AD are included in advance care planning (ACP). The ultimate goal of these documents is to respect patient's autonomy, to facilitate shared goals of care by participating in the decision-making process, including EOL care, and improve patients' quality of life [4].

In Korea, attitudes toward discussion of EOL care or AD may become more favorable according to previous surveys conducted among patients with cancer, their families, oncologists, and the general public $[5,6]$. Community-dwelling elderly individuals have positive attitudes toward AD, and most participants completed the Korean-AD model [6]. However, concerns remain regarding the application of an AD or POLST process in actual clinical practice. First, patients are often excluded from the loop of communication of bad news and the decision-making process, which has predominantly occurred between physicians and family members. Furthermore, EOL discussions are delayed until the very EOL when patients rapidly deteriorate and even unaware of their impending death [7-9]. For those reasons, EOL discussion mostly involves family members, and few patients complete an AD or POLST by themselves in hospital-based studies [9,10]. In addition, lack of knowledge, emotional discomfort of talking about death, or systematic complexity of the process could be other potential barriers.

In this study, we aimed to evaluate the feasibility of discussing POLST in actual oncologic practice. Physicians' and patients' barriers were also explored, which could be informative in finding systematic solutions that facilitate the process of POLST documentation.

\section{Materials and Methods}

\section{Participants}

We designed a prospective, multicenter study that included medical oncologists from seven general hospitals. Eligibility criteria were patients aged $\geq 20$ years with terminal cancer. Terminal cancer is defined as follows: life expectancy of less than several months due to cancer progression despite anticancer treatment or irreversible deterioration of performance status or organ function due to cancer progression [11]. Patients were excluded if they could not sufficiently communicate due to severe illness or cognitive impairment.

\section{Data collection and procedures}

First, the physician in charge tried to introduce the concept of the POLST form to eligible patients. If the form was not formally introduced for any reasons, the questionnaire about physicians' barriers was completed by the physician. Patients who were informed about the form but declined further discussion were asked to complete a questionnaire about patients' barriers. Patients who agreed to discuss the POLST form received detailed explanations and completed the form with the physicians. If the patient declined to complete the form after discussion with the physician, they were also asked to complete the patients' barrier questionnaire.

The POLST form included the following themes: information about the current status of the disease and treatment options; decision-making for the use of LST; decisions concerning hospice care; and information on processes for completion, registration, storage, modification, and withdrawal of the form. LST issues included the use of cardiopulmonary resuscitation $(\mathrm{CPR})$, artificial ventilation, hemodialysis, and anticancer treatment at the EOL. The POLST form was completed if the patient signed the form with confirmation from the physician.

The above procedure was repeated if any significant changes occurred in patients' conditions or personal circumstances. Demographic data, including age, sex, Eastern Cooperative Oncology Group (ECOG) performance status, diagnosis, status of care, follow-up status, and life expectancy were collected. 


\section{Statistical analysis}

The primary endpoint was completion rate of the POLST form defined by the number of patients completed the form divided by the total number of eligible patients. Secondary endpoints were identifying barriers of discussing the POLST. The number of patients was calculated based on the assumption that five patients with terminal cancer are expected to visit each hospital monthly, which estimated that approximately 300 patients would be eligible from the date of institutional review board (IRB) approval until the end of 2017.

Clinical characteristics were presented as counts (percentage) for categorical variables and as median values (range) or mean \pm standard deviation for continuous variables. Descriptive analysis was used to assess barriers for POLST use.

\section{Ethical statement}

This study was approved by the Institutional Review Board of Ulsan University Hospital with a waiver of informed consent (UUH IRB No. 2017-05-003) and performed in accordance with the principles of the Declaration of Helsinki.

\section{Results}

\section{Patient characteristics}

From June to December 2017, 336 patients were eligible. The clinical characteristics of the patients are summarized in Table 1. Median age was 66 years (range, 20 to 94 years), 177 $(52.7 \%)$ were male, and $203(60.4 \%)$ displayed ECOG 3 or 4 performance status. The primary cancer site was hepato-pancreato-biliary $(26.2 \%)$, followed by lung $(23.2 \%)$, gastrointestinal $(19.9 \%)$, and breast $(10.1 \%)$. The others included brain tumor, leukemia, malignancy with unknown primary, soft tissue sarcoma, neuroendocrine tumor, multiple myeloma, skin cancer, and thyroid cancer. At the time of enrollment, 138 patients $(41.2 \%)$ were in hospice care, and 127 $(37.9 \%)$ showed disease progression after anticancer treatment. Remaining patients were still under active treatment $(15.8 \%)$ or were initially diagnosed as terminal cancer $(5.1 \%)$. More than half of patients were recently transferred from other departments $(27.3 \%)$ or hospitals $(30.8 \%)$, with a median follow-up duration of 90 days (range, 1 to 4,046 days). The mean life expectancy of patients, as estimated by their physicians-in-charge, was $10.6 \pm 7.3$ weeks.
Table 1. Baseline characteristics of the eligible patients

\begin{tabular}{|lc} 
Characteristic & $\begin{array}{c}\text { No. of patients } \\
(\mathbf{n}=336)\end{array}$ \\
\hline Age, median (range, yr) & $66(20-94)$ \\
\hline Sex & $177(52.7)$ \\
\hline Male & $159(47.3)$ \\
\hline Female & \\
\hline ECOG & $133(39.6)$ \\
\hline $1 / 2$ & $203(60.4)$ \\
\hline / 4 & \\
\hline Diagnosis & $88(26.2)$ \\
\hline Hepato-pancreato-biliary cancer & $78(23.2)$ \\
\hline Lung cancer & $67(19.9)$ \\
\hline Gastrointestinal cancer & $34(10.1)$ \\
\hline Breast cancer & $26(7.7)$ \\
\hline Genitourinary tract cancer & $19(5.7)$ \\
\hline Head and neck cancer & $24(7.1)$ \\
\hline Others & \\
\hline Treatment status & $17(5.1)$ \\
\hline Initially diagnosed with terminal cancer & $53(15.8)$ \\
\hline Under anticancer treatment & $127(37.8)$ \\
\hline After disease progression & $138(41.1)$ \\
\hline Hospice care & $140(41.9)$ \\
\hline Follow-up status & $91(27.3)$ \\
\hline During follow-up & $103(30.8)$ \\
\hline Transferred from other departments & $10.6 \pm 7.3$ \\
\hline Transferred from other hospitals & \\
\hline Life expectancy (wk) & \\
\hline
\end{tabular}

ECOG, Eastern Cooperative Oncology Group.

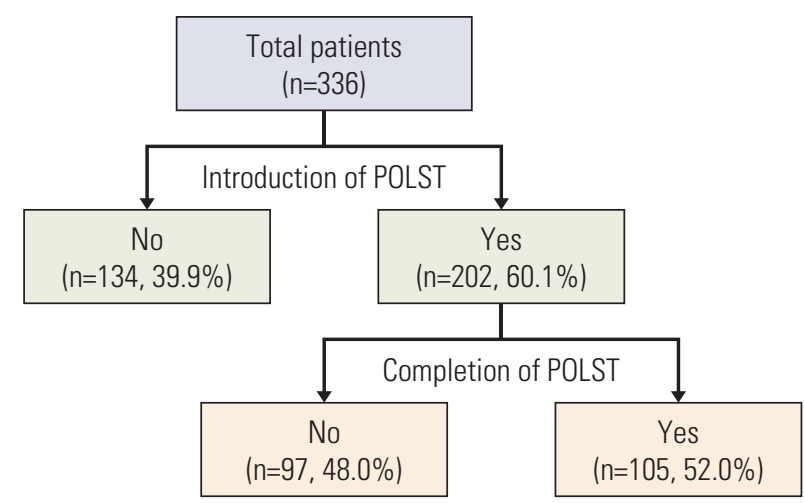

Fig. 1. Patients' diagram. POLST, Physician's Order for Life-sustaining Treatment. 
Table 2. Physicians' barriers

\begin{tabular}{ll} 
Type of barrier & $\begin{array}{c}\text { No. (\%) } \\
(\mathbf{n}=\mathbf{1 8 1})\end{array}$ \\
\hline $\begin{array}{l}\text { Families' reluctance and/or conflict } \\
\text { Lack of rapport with patients }\end{array}$ & $90(49.7)$ \\
\hline Patients' denial or avoidance of the prognosis & $81(44.8)$ \\
\hline Lack of time for discussing the POLST & $62(34.3)$ \\
\hline $\begin{array}{l}\text { Fear of depriving patients' hope or } \\
\text { of abandonment }\end{array}$ & $31(22.7)$ \\
\hline Uncertainty of cancer prognostication & $39(21.5)$ \\
\hline Uncertainty of the right time for POLST discussion & $30(16.6)$ \\
\hline
\end{tabular}

POLST, Physician's Order for Life-sustaining Treatment.

Table 3. Patients' barriers

\begin{tabular}{|c|c|}
\hline Type of barrier & $\begin{array}{l}\text { No. }(\%) \\
(n=63)\end{array}$ \\
\hline Lack of knowledge/Understanding & $41(65.1)$ \\
\hline Lack of knowledge about POLST & $26(41.3)$ \\
\hline Lack of understanding of the POLST paradigm & $26(41.3)$ \\
\hline Emotional discomfort & $40(63.5)$ \\
\hline $\begin{array}{l}\text { Fear of giving-up or being abandoned by } \\
\text { their physicians }\end{array}$ & $19(30.2)$ \\
\hline $\begin{array}{l}\text { Fear of bad things happening after } \\
\text { a POLST documentation }\end{array}$ & $28(44.4)$ \\
\hline Discomfort in talking about death & $19(30.2)$ \\
\hline Refusal or difficulty in decision-making & $42(66.7)$ \\
\hline Trust for family as a surrogate decision-maker & $28(44.4)$ \\
\hline $\begin{array}{l}\text { Trust for a physician as a surrogate } \\
\text { decision-maker }\end{array}$ & $22(34.9)$ \\
\hline Denial of prognosis & $9(14.3)$ \\
\hline
\end{tabular}

POLST, Physician's Order for Life-sustaining Treatment.

\section{Process of POLST}

POLST forms were introduced to 202 patients (60.1\%), of which $105(52.0 \%)$ signed the form (Fig. 1). Among all eligible patients $(n=336)$, the completion rate of the POLST form was $31.3 \%$. For the 202 patients who started to discuss the POLST, the number of discussions was one $(84.7 \%)$, two (13.3\%), and three $(2.0 \%)$. The median time required for discussions was 35 minutes (range, 5 to 120 minutes). Most patients who completed the POLST refused LST at EOL: CPR (98.1\%), artificial ventilation $(99.1 \%)$, hemodialysis $(98.1 \%)$, and anticancer treatment $(91.4 \%)$. Seventy-four patients $(89.2 \%)$ agreed to hospice care. There was no difference of POLST completion rate between hospice patients $(n=43,31.2 \%)$ and non-hospice patients $(\mathrm{n}=62,31.3 \%, \mathrm{p}=0.976)$.

\section{Physicians' barrier for introducing the POLST}

Of the 134 patients that were not introduced to the POLST by the physicians, the questionnaires about the physicians' barriers were completed for 109 cases. Table 2 shows barriers to introduction of POLST from the physicians' perspectives. The major barrier was reluctance or conflict within family members (49.7\%). Family members were unwilling for patients to engage in EOL discussion or conflicts among family members existed for engaging in such discussions. The second most common barrier was the lack of rapport with patients for discussing sensitive issues, such as EOL care and death (44.8\%). Physicians also expressed guilty feelings, such as depriving patients of hope or giving up on patients' care $(21.5 \%)$. Patients' denial or avoidance of their prognosis $(34.3 \%)$, lack of sufficient time in discussing the POLST $(22.7 \%)$, uncertainty of cancer prognostication $(21.0 \%)$, or right time for having such a discussion (16.6\%) were also identified as common barriers.

\section{Patient's barrier in completing the POLST}

Of the 97 patients who declined to complete the POLST, 63 completed the questionnaire about the patients' barrier (Table 3). The most frequently reported patient's barrier was decisional difficulty $(66.7 \%)$, lack of knowledge/ understanding $(65.1 \%)$ of the POLST, and emotional discomfort $(63.5 \%)$. Lack of knowledge or understanding of the POLST was the main barrier: 21 patients $(41.3 \%)$ reported difficulty in understanding the terms or process of the POLST or LST; 21 $(41.3 \%)$ patients had difficulty in understanding the POLST paradigm. Emotional discomfort was also a common barrier for 40 patients $(63.5 \%)$. Specifically, 19 patients $(29.7 \%)$ reported concerns that signing the document considered as giving-up of treatment or being abandoned by their physicians; $28(43.8 \%)$ reported fear of bad things happening after the POLST documentation; $19(29.7 \%)$ felt anxious when talking about death. Forty-two patients $(66.7 \%)$ did not want to make EOL decision by themselves but want their families or doctors to make the decision for them $(44.4 \%$ and $34.9 \%$, respectively). Nine patients $(14.3 \%)$ stated that their disease was not severe enough to discuss the form (not yet, not at this point, or not sick enough). None of the patients answered that they did not trust their physicians. 


\section{Discussion}

In this study, we assessed the feasibility of the POLST documentation for patients with terminal cancer in actual oncologic practice. Approximately one-third of patients completed the POLST form, and various barriers were identified from both physicians' and patients' perspectives.

Numerous studies have assessed the use of ACP or AD, but POLST use has been less commonly studied. Particularly, reports about the preferences for LST including 'do not resuscitate' were found in few studies that examined ACP or $\mathrm{AD}$ [12-14], and even fewer were documented by physicians $[12,15]$. To date, the POLST form is widely accepted in some states in the United States, where completion rate for the POLST form is much higher than that reported in current and previous studies $[16,17]$. In line with the growing interest in patient's autonomy in Korea, recent studies have shown that patients are becoming more involved in EOL discussions $(35.5 \%-55.6 \%)[18,19]$. However, most studies have been retrospective or cross-sectional studies without intervention, or they included too limited or broad populations.

To the best of our knowledge, this study is the first prospective, multicenter study that assesses the feasibility of completing the POLST form for patients with terminal cancer in Korea. The strength of our study is that a wide range of patients with terminal cancer was included: patients with various cancer types; treatment status ranging from patients recently diagnosed with cancer to patients receiving hospice cares; and follow-up status from new patients to patients with long-term follow-up. Seven hospitals from various regions participated, and a consistent process for POLST administration was followed. Considering that our study reflected the real oncologic situation in encountering patients with terminal cancer, a completion rate of $31.3 \%$ suggests that the POLST process may be applicable to patients with terminal cancer.

A variety of barriers of discussing the POLST form emerged from the perspectives of both physicians and patients in this study. The POLST was not even introduced in $39.9 \%$ of patients. From the physician's perspectives, reluctance of family members was the most common barrier to introduce the form. This reluctance was more frequent in this study than in previous studies conducted in Western countries [20-22]. Family-centered culture in Eastern countries including Korea, in which family makes important decisions about a patient's care, could be possible reason [8]. Consistent with previous reports $[20,22,23]$, emotional discomfort for discussing the POLST was also a common barrier for physicians. In the Korean society where talking about death or dying is considered taboo, physicians have difficulty in delivering bad news or having POLST discussions directly with their patients. The belief that patients are not willing to participate in EOL discussion, fear of destroying patients' hope, fear of losing their patients, or lack of longstanding relationships with the patients were primary emotional barriers. Patient's denial of their disease prognosis, uncertainty about prognostication, or right time for POLST discussion was also important barriers $[13,20,23]$. Despite the fact that the prognosis of cancer is relatively predictable compared with other non-cancerous disease [23] and that hematooncology specialists participated in the study, it is still challenging to determine the exact prognosis and right time discussing the POLST.

From the patients' perspectives, lack of knowledge or poor understanding of the POLST was the major barrier for its completion, consistent with previous studies $[13,19,20,24]$. Patients had difficulty in understanding the POLST, such as concept, the process, and advantages and disadvantages. Emotional discomfort for discussing the POLST was also an important barrier, which was also consistent with previous reports $[19,21,23]$. Patients showed fear of being abandoned by their physicians and fear of losing hope or experiencing unfavorable events if they sign the POLST form. In addition, some patients preferred letting their family or doctors make decisions for their future medical care. These barriers might reflect emotional discomfort related to EOL discussions or lack of capacity to make decisions for EOL medical care. In Eastern countries, people favor making EOL decisions with their family members [25].

Systematic barriers, including lack of infrastructure (time, space, trained human resources, or systemic support), were also important in previous studies [20,21,26,27]. Our study also revealed lack of time as a barrier. The median time required for the POLST discussion was 35 minutes, which is significantly longer than the average outpatient consultation time of 5 minutes in Korea [28].

These barriers are interrelated and should not be interpreted in isolation. Means of overcoming barriers might be also interconnected. First, public engagement in an open discussion about death or dying should become more natural in Korean society to relieve the emotional barrier. Evidence shows that patients expect their physicians to initiate an honest and open conversation about their disease and prognosis $[7,21,25]$. Simply being asked to discuss AD or POLST facilitates such a process [12,15]. In fact, half of the patients in this study completed the POLST document after being asked. Unfortunately, health-care providers, including physicians, are not adequately trained for delivery of bad news and EOL communication. Therefore, education regarding communication skills among health-care providers who engage in EOL discussion is extremely important [29]. Professional training may ease emotional and technical difficulties associated with EOL discussions. 
Further, patients want to know what ensues after completing the POLST form. Detailed explanations about palliative care to relieve suffering might be helpful in decreasing emotional barriers of patients [21]. Since lack of knowledge was the main barrier, public awareness or educational campaigns using decision support tools are needed to increase knowledge in the general public about EOL discussions [30].

Third, designation of surrogates who can make EOL decisions for patients could enhance the completion rate of the POLST document. Designation of surrogates is a major component of ACP, AD, and POLST, and surrogates participate in POLST documentation in other countries, though not in Korea. Our study shows that major barriers included reluctance of family members, physicians, and patients to directly discuss this sensitive issue with patients. In addition, some terminal cancer patients could not initiate the POLST discussion because of physical or mental insufficiency. Although patients' autonomy is an important aspect of the POLST form, designation of surrogates can indirectly reflect the best interests of patients.

The limited time of physicians is also a challenging barrier. Systemic support, such as simplifying the process, involving trained non-physician facilitators, and being compensated for health-care costs, might help overcome this barrier [27].

This study had limitations. First, some selection bias existed for overestimating results. Hemato-oncology specialists who might be more familiar and experienced with EOL discussions participated in this study. Few large tertiary hospitals focusing on active anticancer treatment participated. In addition, we included patients who were capable of communicating, which excluded the majority of patients with terminal cancer because of poor performance status and inability to communicate. A considerable number of patients had already chosen hospice care. Although the completion rate of POLST was not different between hospice and non-hospice patients in our study, caution should be taken when generalizing our results. Second, some physicians and patients did not complete the questionnaire, and the barriers from the family members' perspectives were not investigated. Third, we focused on the completion of the POLST but not evaluated the qualities or outcomes of the documents.

In conclusions, one-third of patients with terminal cancer completed the POLST, and a wide range of barriers from physicians, families, patients, and systematic aspects were identified. These were interconnected, and there are rooms for the improvement of the POLST discussion. Further studies need to be conducted to investigate the quality or outcomes of the POLST decision in the future.

\section{Conflicts of Interest}

Conflict of interest relevant to this article was not reported.

\section{Acknowledgments}

The research was supported by the Korean Cancer Study Group (KCSG PC17-01).

\section{References}

1. Korean Supreme Court. Withdrawing futile life-sustaining treatment. Seoul: Korean Supreme Court; 2009.

2. Ministry of Health and Welfare. Act on hospice and palliative care and decisions on life-sustaining treatment for patients at the end of life. Sejong: Ministry of Health and Welfare; 2016.

3. Royal College of Physicians. Advance care planning: concise guideline to good practice, No. 12. London: Royal College of Physicians; 2009.

4. Houben CH, Spruit MA, Groenen MT, Wouters EF, Janssen DJ. Efficacy of advance care planning: a systematic review and meta-analysis. J Am Med Dir Assoc. 2014;15:477-89.

5. Keam B, Yun YH, Heo DS, Park BW, Cho CH, Kim S, et al. The attitudes of Korean cancer patients, family caregivers, oncologists, and members of the general public toward advance directives. Support Care Cancer. 2013;21:1437-44.

6. Kim S, Hong SW, Kim J. Feasibility of the Korean-advance directives among community-dwelling elderly persons. Holist Nurs Pract. 2017;31:234-42.
7. Yun YH, Lee CG, Kim SY, Lee SW, Heo DS, Kim JS, et al. The attitudes of cancer patients and their families toward the disclosure of terminal illness. J Clin Oncol. 2004;22:307-14.

8. Heo DS. Patient autonomy and advance directives in Korea. J Korean Med Assoc. 2009;52:865-70.

9. Lee JK, Keam B, An AR, Kim TM, Lee SH, Kim DW, et al. Surrogate decision-making in Korean patients with advanced cancer: a longitudinal study. Support Care Cancer. 2013;21:183-90.

10. Kwon SH, Im SH, Cho KW, Cho E, Yoon SJ, Oh SY. Most advance directives written by patients with advanced cancer or their proxies request only minimally invasive treatments during end-of-life care. Am J Hosp Palliat Care. 2012;29: 622-6.

11. Lee SM, Kim SJ, Choi YS, Heo DS, Baik S, Choi BM, et al. Consensus guidelines for the definition of the end stage of disease and last days of life and criteria for medical judgment. J Korean Med Assoc. 2018;61:509-21.

12. Alano GJ, Pekmezaris R, Tai JY, Hussain MJ, Jeune J, Louis B, 
et al. Factors influencing older adults to complete advance directives. Palliat Support Care. 2010;8:267-75.

13. McDonald JC, du Manoir JM, Kevork N, Le LW, Zimmermann C. Advance directives in patients with advanced cancer receiving active treatment: attitudes, prevalence, and barriers. Support Care Cancer. 2017;25:523-31.

14. Salmond SW, David E. Attitudes toward advance directives and advance directive completion rates. Orthop Nurs. 2005; 24:117-27.

15. Phipps E, True G, Harris D, Chong U, Tester W, Chavin SI, et al. Approaching the end of life: attitudes, preferences, and behaviors of African-American and white patients and their family caregivers. J Clin Oncol. 2003;21:549-54.

16. Lammers AJ, Zive DM, Tolle SW, Fromme EK. The oncology specialist's role in POLST form completion. Am J Hosp Palliat Care. 2018;35:297-303.

17. Tarzian AJ, Cheevers NB. Maryland's medical orders for lifesustaining treatment form use: reports of a statewide survey. J Palliat Med. 2017;20:939-45.

18. Kong BH, An HJ, Kim HS, Ha SY, Kim IK, Lee JE, et al. Experience of advance directives in a hospice center. J Korean Med Sci. 2015;30:151-4.

19. Kim S, Koh S, Park K, Kim J. End-of-life care decisions using a Korean advance directive among cancer patient-caregiver dyads. Palliat Support Care. 2017;15:77-87.

20. You JJ, Downar J, Fowler RA, Lamontagne F, Ma IW, Jayaraman D, et al. Barriers to goals of care discussions with seriously ill hospitalized patients and their families: a multicenter survey of clinicians. JAMA Intern Med. 2015;175:549-56.

21. Simon J, Porterfield P, Bouchal SR, Heyland D. 'Not yet' and 'Just ask': barriers and facilitators to advance care planning: a qualitative descriptive study of the perspectives of seriously ill, older patients and their families. BMJ Support Palliat Care. 2015;5:54-62.
22. Travers A, Taylor V. What are the barriers to initiating endof-life conversations with patients in the last year of life? Int J Palliat Nurs. 2016;22:454-62.

23. De Vleminck A, Pardon K, Beernaert K, Deschepper R, Houttekier D, Van Audenhove C, et al. Barriers to advance care planning in cancer, heart failure and dementia patients: a focus group study on general practitioners' views and experiences. PLoS One. 2014;9:e84905.

24. Hubert E, Schulte N, Belle S, Gerhardt A, Merx K, Hofmann WK, et al. Cancer patients and advance directives: a survey of patients in a hematology and oncology outpatient clinic. Onkologie. 2013;36:398-402.

25. Ivo K, Younsuck K, Ho YY, Sang-Yeon S, Seog HD, Hyunah $\mathrm{B}$, et al. A survey of the perspectives of patients who are seriously ill regarding end-of-life decisions in some medical institutions of Korea, China and Japan. J Med Ethics. 2012;38:310-6.

26. Hagen NA, Howlett J, Sharma NC, Biondo P, Holroyd-Leduc J, Fassbender K, et al. Advance care planning: identifying system-specific barriers and facilitators. Curr Oncol. 2015;22:e23745.

27. Kirchhoff KT, Hammes BJ, Kehl KA, Briggs LA, Brown RL. Effect of a disease-specific advance care planning intervention on end-of-life care. J Am Geriatr Soc. 2012;60:946-50.

28. Lee CH, Lim H, Kim Y, Park AH, Park EC, Kang JG. Analysis of appropriate outpatient consultation time for clinical departments. Health Policy Manag. 2014;24:254-60.

29. Back AL, Arnold RM, Baile WF, Fryer-Edwards KA, Alexander SC, Barley GE, et al. Efficacy of communication skills training for giving bad news and discussing transitions to palliative care. Arch Intern Med. 2007;167:453-60.

30. Butler M, Ratner E, McCreedy E, Shippee N, Kane RL. Decision aids for advance care planning: an overview of the state of the science. Ann Intern Med. 2014;161:408-18. 